\title{
UNSYMMETRIC SOLUTIONS FOR THE CYLINDRICAL MEMBRANE
}

\author{
BY
}

R. W. DICKEY

University of Wisconsin, Madison

1. Introduction. The purpose of this paper is to discuss both the symmetric and unsymmetric behavior of the cylindrical membrane in both the presence and absence of a constant normal pressure. We will adopt the approximate theory suggested by Von Karman and Tsien [1] for cylindrical shells. For the membrane these equations simplify to

$$
\begin{gathered}
\varepsilon_{x}=\frac{\partial u}{\partial x}+\frac{1}{2}\left(\frac{\partial w}{\partial x}\right)^{2} \\
\varepsilon_{s}=\frac{w}{a}+\frac{\partial v}{\partial s}+\frac{1}{2}\left(\frac{\partial w}{\partial s}\right)^{2} \\
\varepsilon_{x s}=\frac{1}{2}\left(\frac{\partial u}{\partial s}+\frac{\partial v}{\partial x}\right)+\frac{1}{2} \frac{\partial w}{\partial x} \frac{\partial w}{\partial s} \\
\sigma_{x}=\frac{E}{1-\nu^{2}}\left(\varepsilon_{x}+\nu \varepsilon_{s}\right) \\
\sigma_{s}=\frac{E}{1-\nu^{2}}\left(\varepsilon_{s}+\nu \varepsilon_{x}\right) \\
\sigma_{x s}=\frac{E}{1-\nu} \varepsilon_{x s} \\
\frac{\partial}{\partial x} \sigma_{x s}+\frac{\partial}{\partial s} \sigma_{s}=0 \\
\frac{\partial}{\partial x} \sigma_{x}+\frac{\partial}{\partial s} \sigma_{x s}=0 \\
\frac{\partial}{\partial x}\left(\sigma_{x} \frac{\partial w}{\partial x}+\sigma_{x s} \frac{\partial w}{\partial s}\right)+\frac{\partial}{\partial s}\left(\sigma_{s} \frac{\partial w}{\partial s}+\sigma_{x s} \frac{\partial w}{\partial x}\right)-\frac{\sigma_{s}}{a}+\frac{P}{h}=0 .
\end{gathered}
$$

The equations (1.1) are the strain-displacement equations, (1.2) are the constitutive equations, and (1.3) are the equilibrium equations. The quantities $\sigma_{x}, \sigma_{s}$, and $\sigma_{x s}$ are the

*Received October 23, 1985. 
axial, circumferential, and shear stresses, and similarly $\varepsilon_{x}, \varepsilon_{s}$, and $\varepsilon_{x s}$ are the axial, circumferential, and shear strains. The normal, axial, and circumferential displacements are $w(x, s), u(x, s), v(x, s) . P$ is the constant normal pressure, $h$ is the thickness of the membrane, $E$ is the Young's modulus, and $\nu$ is the Poisson ratio. In the cylindrical coordinate system the $x$-axis is the axis of symmetry of the undeformed cylinder and $s$, the circumferential coordinate, is taken around the circumference perpendicular to the generator of the cylinder wall. In the case of axially symmetric deformations, the equations (1.1), (1.2), and (1.3) reduce to the theory discussed by Bromberg and Stoker [2] and Reissner [3].

For simplicity we will assume that the deformations are symmetric about the center of the cylinder. If we denote the length of the cylinder by $2 l$ and the radius by $a$, the boundary conditions are

$$
\begin{gathered}
\frac{\partial w(0, s)}{\partial x}=w(l, s)=0, \\
\frac{\partial v(0, s)}{\partial x}=v(l, s),
\end{gathered}
$$

and either the stress condition

$$
\frac{\partial \sigma_{x}(0, s)}{\partial x}=0, \quad \sigma_{x}(l, s)=S,
$$

or the displacement condition

$$
u(0, s)=0, \quad u(l, s)=\mu .
$$

In addition we require all of the dependent variables to be periodic in $s$ with period $2 \pi a$.

In Section 2 we introduce the Airy stress function in order to reduce the system of partial differential equations (1.1), (1.2), and (1.3) to a pair of nonlinear partial differential equations. In Section 3 the radially symmetric case is discussed for both $S>0$ and $S<0$ and for $\mu>0$ and $\mu<0$. This symmetric case is elementary and can be solved exactly. However, the cases $s<0$ and $\mu<0$ are interesting since there are infinitely many values of $s<0$ for which there is no symmetric solution and for each value of $\mu<0$ there are infinitely many symmetric solutions. In order to study the unsymmetric solutions the nonlinear equations are linearized about the known symmetric solutions. In Section 4 the existence of nontrivial solutions to this homogeneous linear system is studied. Even though the system is linear it has some very unpleasant features. In particular, the system has periodic coefficients which become highly oscillatory as $S \rightarrow 0(S<0)$, and in addition the system reduces in order as $S \rightarrow 0$. However, there is one choice of the applied pressure $P$ (as a function of $S$ ) for which the system can be solved exactly. In Section 4 it is shown how to use this fact to study the case $P=0$. A method is described which enables us to determine approximate values of $S$ for which unsymmetric solutions bifurcate from the symmetric solutions and the form of these solutions is also determined. The results indicate that there is an infinite sequence of values $S_{n} \rightarrow 0\left(S_{n}<0\right)$ for which unsymmetric solutions bifurcate from the symmetric solutions. 
2. Airy stress function. It is convenient to simplify equations (1.1), (1.2), and (1.3) by introducing the Airy stress function $\varphi(x, s)$. The function $\varphi$ is defined by

$$
\sigma_{s}=\frac{\partial^{2} \varphi}{\partial x^{2}}, \quad \sigma_{x}=\frac{\partial^{2} \varphi}{\partial s^{2}}, \quad \sigma_{x s}=-\frac{\partial^{2} \varphi}{\partial x \partial s} .
$$

The equations (1.1), (1.2), and (1.3) reduce to a pair of nonlinear partial differential equations for the determination of $\varphi(x, s)$ and $w(x, s)$,

$$
\begin{gathered}
\frac{\partial^{2} \varphi}{\partial s^{2}} \frac{\partial^{2} w}{\partial x^{2}}-\frac{\partial^{2} \varphi}{\partial x \partial s} \frac{\partial^{2} w}{\partial x \partial s}+\frac{\partial^{2} \varphi}{\partial x^{2}} \frac{\partial^{2} w}{\partial s^{2}}-\frac{1}{a} \frac{\partial^{2} \varphi}{\partial x^{2}}+\frac{P}{h}=0, \\
\Delta^{2} \varphi=E\left\{\left(\frac{\partial^{2} w}{\partial x \partial s}\right)^{2}-\frac{\partial^{2} w}{\partial x^{2}} \frac{\partial^{2} w}{\partial s^{2}}+\frac{1}{a} \frac{\partial^{2} w}{\partial x^{2}}\right\}
\end{gathered}
$$

where

$$
\Delta=\frac{\partial^{2}}{\partial x^{2}}+\frac{\partial^{2}}{\partial s^{2}}
$$

In order to determine the boundary conditions on (2.2) we note that (1.1b) and (1.4) imply

$$
\frac{\partial \varepsilon_{s}(0, s)}{\partial x}=\varepsilon_{s}(l, s)=0 .
$$

Equations (1.2a), (1.2b), and (2.1) yield

$$
\varepsilon_{s}=\frac{1}{E}\left(\sigma_{s}-\nu \sigma_{x}\right)=\frac{1}{E}\left(\frac{\partial^{2} \varphi}{\partial x^{2}}-\nu \frac{\partial^{2} \varphi}{\partial s^{2}}\right) .
$$

Equations (1.4c), (2.1), (2.4), and (2.5) imply the stress boundary conditions

$$
\begin{gathered}
\frac{\partial}{\partial x}\left(\frac{\partial^{2} \varphi(0, s)}{\partial s^{2}}\right)=0, \quad \frac{\partial^{2} \varphi(l, s)}{\partial s^{2}}=S, \\
\frac{\partial^{3} \varphi(0, s)}{\partial x^{3}}=0, \quad \frac{\partial^{2} \varphi(l, s)}{\partial x^{2}}=\nu S .
\end{gathered}
$$

The boundary condition on $w$ is given by (1.4a).

The displacement condition is more complicated and will be discussed later.

3. Symmetric solutions. Equations (2.2) have special solutions of the form

$$
\begin{gathered}
w(x, s)=W(x), \\
\varphi(x, s)=\Phi(x)+S s^{2} / 2
\end{gathered}
$$

where $W(x)$ and $\Phi(x)$ must satisfy

$$
\begin{gathered}
S W^{\prime \prime}-\frac{1}{a} \Phi^{\prime \prime}+\frac{P}{h}=0, \\
\Phi^{(4)}=\frac{E}{a} W^{\prime \prime}
\end{gathered}
$$


$\left({ }^{\prime}=d / d x\right)$ and boundary conditions

$$
\begin{gathered}
W^{\prime}(0)=W(l)=0, \\
\Phi^{\prime \prime \prime}(0)=0, \quad \Phi^{\prime \prime}(l)=\nu S .
\end{gathered}
$$

Equation (3.2b) may be integrated, so that

$$
\Phi^{\prime \prime}-\frac{E}{a} W=\nu S .
$$

Equations (3.4) and (3.2a) may be combined to yield a single equation for the determination of $W$,

$$
W^{\prime \prime}-\frac{E}{a^{2} S} W=\frac{\nu}{a}-\frac{P}{S h} .
$$

The nature of the solution of (2.5) depends on the size of $S$. Introduce the notation

$$
\alpha^{2}=\frac{|S|}{E} .
$$

If $S>0$ (Tensile case) the solution of (3.5) satisfying the boundary condtion (3.3a) is

$$
W=\left(\frac{P a^{2}}{E h}-\nu a \alpha^{2}\right)\left(1-\frac{\cosh \left(\frac{x}{a \alpha}\right)}{\cosh \left(\frac{l}{a \alpha}\right)}\right) .
$$

In this case equation (3.4) implies

$$
\Phi^{\prime \prime}=\frac{P a}{h}+\left(\nu E \alpha^{2}-\frac{P a}{h}\right) \frac{\cosh \left(\frac{x}{a \alpha}\right)}{\cosh \left(\frac{l}{a \alpha}\right)} .
$$

It is of interest to note that if $P=0$ then $W \rightarrow 0$ as $\alpha \rightarrow 0$ (i.e., as $S \rightarrow 0$ ). However, if $P \neq 0$ then the limit as $\alpha \rightarrow 0$ is not uniform. It is easily seen that $W \rightarrow P a^{2} / E h$ as $\alpha \rightarrow 0$ when $0 \leqslant x<l$ and $W=0$ when $x=l$.

In addition to the stress problem we would like to find the solution(s) which satisfy the displacement condition (1.4c). It is a consequence of (1.1a), (1.2), and (2.1) that

$$
\frac{1}{E}\left(\frac{\partial^{2} \varphi}{\partial s^{2}}-\nu \frac{\partial^{2} \varphi}{\partial x^{2}}\right)=\frac{\partial u}{\partial x}+\frac{1}{2}\left(\frac{\partial w}{\partial x}\right)^{2} .
$$

In the symmetric case, (3.1) and (3.9) imply

$$
\frac{1}{E}\left(S-\nu \Phi^{\prime \prime}\right)=\frac{\partial u}{\partial x}+\frac{1}{2}\left(W^{\prime}\right)^{2}
$$

where $\Phi^{\prime \prime}$ is given by (3.8) and $W$ is given by (3.7). An integration from $x=0$ to $x=l$ yields

$$
\begin{aligned}
\frac{\mu}{l}=\alpha^{2}-\nu\left\{\frac{P a}{E h}\right. & \left.+\frac{a \alpha}{l}\left(\nu \alpha^{2}-\frac{P a}{E h}\right) \tanh \left(\frac{l}{a \alpha}\right)\right\} \\
& -\frac{1}{2 \alpha}\left(\frac{P a}{E h}-\nu \alpha^{2}\right)^{2}\left(\frac{\frac{a \alpha}{l} \sinh \left(\frac{2 l}{a \alpha}\right)-2}{4 \cosh ^{2}\left(\frac{l}{a \alpha}\right)}\right) .
\end{aligned}
$$


A sketch of (3.11) is given in Figure 1. Of course an actual graph of (3.11) depends on the values of $l / a, P a / E h$, and $\nu$. However, the qualitative picture is not sensitive to changes in these quantities (the curve in Figure 1 was actually calculated for a wide variety of these parameters). It is clear from Figure 1 that in the tensile case, symmetric solutions to the displacement problem are unique.

If $S<0$ (compressive case) the behavior changes. The solution of (3.5) satisfying the boundary condition $(3.3 \mathrm{a})$ is

$$
W=\left(\nu a \alpha^{2}+\frac{P a^{2}}{E h}\right)\left(1-\frac{\cos \left(\frac{x}{a \alpha}\right)}{\cos \left(\frac{l}{a \alpha}\right)}\right)
$$

if $l /(a \alpha) \neq(2 k+1) \pi / 2(k=0,1,2, \ldots)$. If $l /(\alpha a)=(2 k+1) \pi / 2$ for some integer $k$ there is no solution. Equation (3.4) implies

$$
\Phi^{\prime \prime}=\frac{P a}{h}-\left(\nu E \alpha^{2}+\frac{P a}{h}\right) \frac{\cos \left(\frac{x}{\alpha a}\right)}{\cos \left(\frac{l}{\alpha a}\right)} .
$$
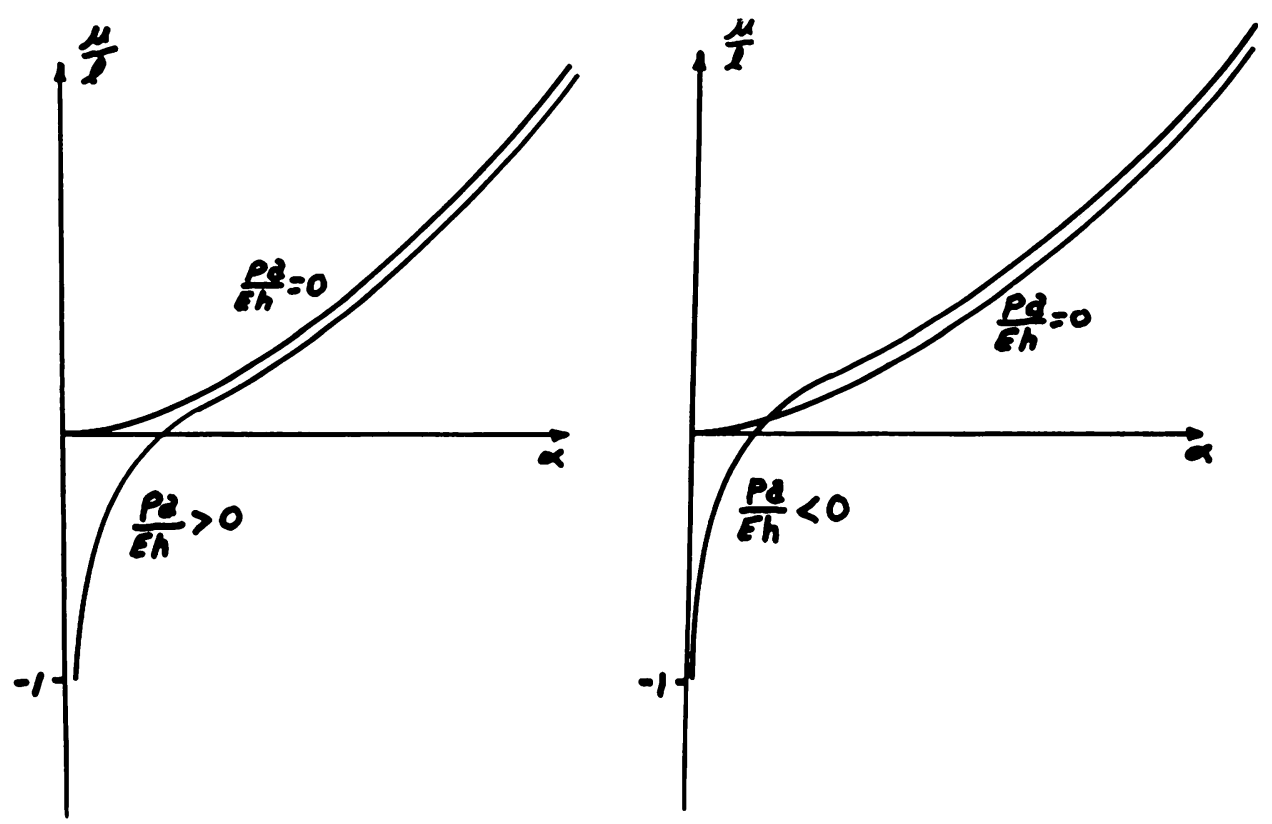

FIG. 1. 
In addition equation (3.10) implies that in the compressive case

$$
\begin{aligned}
\frac{\mu}{l}=-\alpha^{2}-\nu\left\{\frac{P a}{E h}\right. & \left.+\frac{a \alpha}{l}\left(\nu \alpha^{2}+\frac{P a}{E h}\right) \tan \left(\frac{l}{\alpha a}\right)\right\} \\
& -\frac{1}{2 \alpha}\left(\nu \alpha^{2}+\frac{P a}{E h}\right)^{2}\left(\frac{\frac{a \alpha}{l} \sin \left(\frac{2 l}{a \alpha}\right)+2}{4 \cos ^{2}\left(\frac{l}{a \alpha}\right)}\right) .
\end{aligned}
$$

A sketch of (3.14) is given in Figure 2. Note that (3.14) is defined in a sequence of intervals $(2 k-1) \pi / 2<l /(\alpha a)<(2 k+1) \pi / 2(k=1,2, \ldots)$. Thus (3.14) has infinitely many branches converging to zero as $\alpha \rightarrow 0$. In addition the maximum value of $\mu / l$ in the interval $(2 k-1) \pi / 2<l /(\alpha a)<(2 k+1) \pi / 2$ approaches zero as $k \rightarrow \infty$. Just as in the tensile case the actual value of $\mu / l$ for a given value of $\alpha$ depends on $l / a, P a /(E h)$, and $\nu$. However, the qualitative picture is unaffected by changes in these parameters.

It is clear from Figure 2 that if a negative value of $\mu$ is specified as a boundary condition then there are infinitely many choices of $\alpha$ yielding that value of $\mu$, and hence infinitely many axially symmetric solutions.

4. Unsymmetric Solutions. It is plausible that, in addition to the symmetric solutions described in Section 3, the cylindrical membrane would have solutions which depend on $s$ -at least in the compressive case. Indeed in the case of the pressure free cylindrical shell this is known. (There is a large literature on this topic. One of the early treatments was

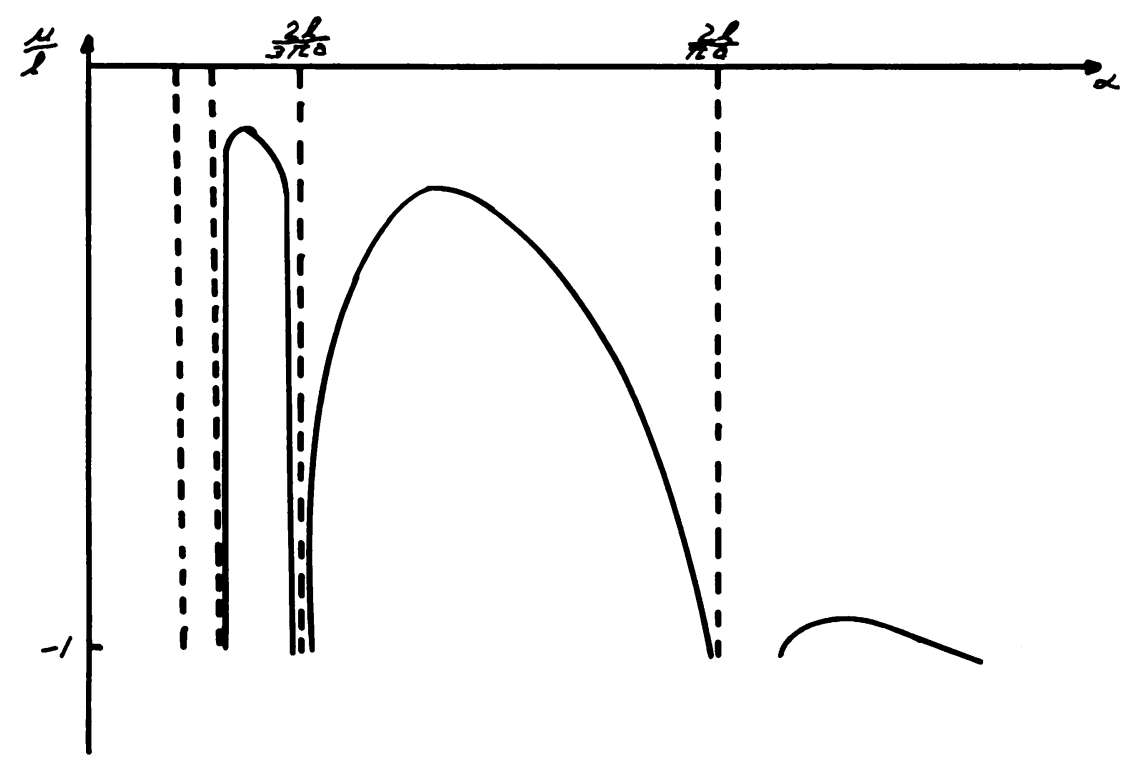

FIG. 2. 
given by von Karman [1].) However, because of the singular nature of the membrane equations the approaches used in shell theory are not appropriate and a different method must be found. In this section we will describe a method for approximating the values of $S$ for which angle-dependent solutions bifurcate from the symmetric solutions described in Section 3.

It is convenient to begin by linearizing the nonlinear equations (2.2) about the exact solution (3.12) and (3.13) and look for nontrivial solutions of these linearized equations.

Thus we introduce

$$
\begin{gathered}
\varphi(x, s)=\Phi(x)+S s^{2} / 2+\Psi(x, s), \\
w(x, s)=W(x)+\omega(x, s),
\end{gathered}
$$

where $\Psi(x, s)$ and $\omega(x, s)$ are small of first order and $W(x)$ and $\Phi(x)$ are given by (3.13) and (3.14). The boundary conditions (2.6) and (2.7) imply that

$$
\begin{gathered}
\frac{\partial}{\partial x}\left(\frac{\partial^{2} \Psi(0, s)}{\partial s^{2}}\right)=\frac{\partial^{2} \Psi(l, s)}{\partial s^{2}}=0, \\
\frac{\partial^{3} \Psi(0, s)}{\partial x^{3}}=\frac{\partial^{2} \Psi(l, s)}{\partial x^{2}}=0, \\
\frac{\partial \omega(0, s)}{\partial x}=\omega(l, s)=0,
\end{gathered}
$$

and all quantities to be periodic in $s$ of period $2 \pi a$.

In order to obtain the linearized equations, place (4.1) and (4.2) in (2.2a) and (2.2b). If we retain only terms which are linear in $\Psi$ and $\omega$ we find

$$
\begin{gathered}
S \omega_{x x}+W^{\prime \prime} \Psi_{s s}+\Phi^{\prime \prime} \omega_{s s}-\frac{1}{a} \Psi_{x x}=0 \\
\Psi_{s s s s}+2 \Psi_{x x s s}+\Psi_{x x x x}=E\left(-W^{\prime \prime} \omega_{s s}+\frac{1}{a} \omega_{x x}\right) .
\end{gathered}
$$

We look for solutions of (4.5) of the form

$$
\begin{aligned}
& \omega(x, s)=\omega(x) e^{i n s / a}, \\
& \Psi(x, s)=\Psi(x) e^{i n s / a} .
\end{aligned}
$$

After this substitution equations (4.5) reduce to the pair of linear ordinary differential equations

$$
\begin{gathered}
S \omega^{\prime \prime}-W^{\prime \prime}\left(\frac{n^{2}}{a^{2}}\right) \Psi-\frac{n^{2}}{a^{2}} \Psi^{\prime \prime} \omega-\frac{1}{a} \Psi^{\prime \prime}=0 \\
\Psi^{(4)}-\frac{2 n^{2}}{a^{2}} \Psi^{\prime \prime}+\frac{n^{4}}{a^{4}} \Psi=E\left(\frac{n^{2}}{a^{2}} W^{\prime \prime} \omega+\frac{1}{a} \omega^{\prime \prime}\right) .
\end{gathered}
$$


Combining (4.7) with (3.12), (3.13) we find

$$
\begin{aligned}
& \frac{1}{a} \Psi^{\prime \prime}+\frac{n^{2}}{a^{3} \alpha^{2}}\left(\nu \alpha^{2}+\frac{P a}{E h} \frac{\cos \left(\frac{x}{\alpha a}\right)}{\cos \left(\frac{l}{\alpha a}\right)} \Psi\right. \\
&\left.=-E \alpha^{2} \omega^{\prime \prime}-E \frac{n^{2}}{a^{2}}\left(\frac{P a}{E h}-\left\{\nu \alpha^{2}+\frac{P a}{E h}\right\}\right) \frac{\cos \left(\frac{x}{\alpha a}\right)}{\cos \left(\frac{l}{\alpha a}\right)}\right) \omega \\
& \Psi^{(4)}-\frac{2 n^{2}}{a^{2}} \Psi^{\prime \prime}+\frac{n^{4}}{a^{4}} \psi=\frac{E}{a} \omega^{\prime \prime}+\frac{E n^{2}}{a^{3} \alpha^{2}}\left(\nu \alpha^{2}+\frac{P a}{E h}\right) \frac{\cos \left(\frac{x}{\alpha a}\right)}{\cos \left(\frac{l}{\alpha a}\right)} \omega
\end{aligned}
$$

The case of most interest is the case $P=0$. For the choice $P=0$ equations (4.8) reduce to

$$
\begin{gathered}
\frac{1}{a} \Psi^{\prime \prime}+\frac{\nu n^{2}}{a^{3}} \frac{\cos \left(\frac{x}{\alpha a}\right)}{\cos \left(\frac{l}{\alpha a}\right)} \Psi=-E \alpha^{2} \omega^{\prime \prime}-\frac{\nu E n^{2} \alpha^{2}}{a^{2}} \frac{\cos \left(\frac{x}{\alpha a}\right)}{\cos \left(\frac{l}{a \alpha}\right)} \omega, \\
\Psi^{(4)}-2 \frac{n^{2}}{a^{2}} \Psi^{\prime \prime}+\frac{n^{4}}{a^{4}} \Psi=\frac{E}{a} \omega^{\prime \prime}+\frac{\nu E n^{2}}{a^{3}} \frac{\cos \left(\frac{x}{\alpha a}\right)}{\cos \left(\frac{l}{\alpha a}\right)} \omega .
\end{gathered}
$$

The boundary conditions on $\Psi(x)$ and $\omega(x)$ are immediately determined from (4.3) and (4.4)

$$
\begin{aligned}
\Psi^{\prime}(0) & =\Psi(l)=0, \\
\Psi^{\prime \prime \prime}(0) & =\Psi^{\prime \prime}(l)=0, \\
\omega^{\prime}(0) & =\omega(l)=0 .
\end{aligned}
$$

We would like to find values of $\alpha$ for which (4.9) has nontrivial solutions satisfying the boundary conditions (4.11). As has been noted above it is to be expected that the problem has nontrivial solutions. However, the solution of (4.9) presents several difficulties. In particular, if bifurcation occurs for small $\alpha$-which is likely-the cosine term in (4.9) becomes highly oscillatory. In addition the equations are singular, i.e., they reduce in order as $\alpha \rightarrow 0$. In order to circumvent this difficulty we note that for the particular choice of the pressure

$$
\frac{P a}{E h}=-\nu \alpha^{2}
$$


the equations reduce to

$$
\begin{gathered}
\frac{1}{a} \Psi^{\prime \prime}=-E \alpha^{2} \omega^{\prime \prime}+\frac{\nu E n^{2} \alpha^{2}}{a^{2}} \omega \\
\Psi^{(4)}-\frac{2 n^{2}}{a^{2}} \Psi^{\prime \prime}+\frac{n^{4}}{a^{4}} \Psi=\frac{E}{a} \omega^{\prime \prime}
\end{gathered}
$$

The pair of equations (4.13) with boundary conditions (4.10) and (4.11) can be solved exactly. The solutions are

$$
\begin{aligned}
& \Psi=A_{k} \cos \left(\beta_{k} \frac{x}{l}\right), \\
& \omega=B_{k} \cos \left(\beta_{k} \frac{x}{l}\right)
\end{aligned}
$$

where

$$
\beta_{k}=\frac{2 k+1}{2} \pi
$$

if $\alpha=\alpha_{k}$ with

$$
\alpha_{k}^{2}=\frac{\gamma^{2} \beta_{k}^{4}}{\left[\beta_{k}^{4}+2 n^{2} \gamma^{2} \beta_{k}^{2}+n^{4} \gamma^{4}\right]\left[\beta_{k}^{2}+\nu n^{2} \gamma^{2}\right]}
$$

when $k=0,1,2, \ldots$ and

$$
\gamma=l / a \text {. }
$$

The coefficients $A_{k}$ and $B_{k}$ are any nontrivial solutions of the pair of algebraic equations

$$
\begin{gathered}
\frac{\beta_{k}^{2}}{a l^{2}} A_{k}+E \alpha_{k}^{2}\left(\frac{\beta_{k}^{2}}{l^{2}}+\frac{\nu n^{2}}{a^{2}}\right) B_{k}=0, \\
\left(\frac{\beta_{k}^{4}}{l^{4}}+2 \frac{n^{2}}{a^{2}} \frac{\beta_{k}^{2}}{l^{2}}+\frac{n^{4}}{a^{4}}\right) A_{k}+\frac{E}{a} \frac{\beta_{k}^{2}}{l^{2}} B_{k}=0 .
\end{gathered}
$$

Thus there are infinitely many pairs $\left(\alpha_{k}, P_{k}\right)$ with

$$
\frac{P_{k} a}{E h}=-\nu \alpha_{k}^{2}
$$

(cf. (4.12)) for which (4.13) with boundary conditions (4.10) and (4.11) have nontrivial solutions. We also note that $\alpha_{k} \rightarrow 0$ as $k \rightarrow \infty$, i.e., $\alpha=0$ is a limit point of eigenvalues of (4.13).

We would expect the solutions, even the angle-dependent solutions for the cylindrical membrane, to depend smoothly on the applied pressure and boundary stress. If this is the case the point $\left(\alpha_{k}, P_{k}\right)$ must be a point on a curve

$$
P=f(\alpha ; k)
$$


of values for which (4.8) with boundary conditions (4.10) and (4.11) have nontrivial solutions. These curves and the corresponding solutions can be computed by perturbations. In particular,

$$
\begin{aligned}
& P=-\frac{\nu E h}{a} \alpha_{k}^{2}+\frac{E h}{a} f_{1}(k) \varepsilon+\cdots \\
& \varepsilon=\alpha-\alpha_{k} \\
& \Psi(x ; k)=\Psi_{0}(x ; k)+\Psi_{1}(x ; k) \varepsilon+\cdots \\
&=A_{k} \cos \left(\beta_{k} \frac{x}{l}\right)+\Psi_{1}(x ; k) \varepsilon+\cdots \\
& \omega(x ; k)=\omega_{0}(x ; k)+\omega_{1}(x ; k) \varepsilon+\cdots \\
&=B_{k} \cos \left(\beta_{k} \frac{x}{l}\right)+\omega_{1}(x ; k) \varepsilon+\cdots .
\end{aligned}
$$

The perturbation scheme indicated for (4.21), (4.22), and (4.23) leads to a series of differential equations. The equations for the determination of $\Psi_{1}(x ; k)$ and $\omega_{1}(x ; k)$ are

$$
\begin{gathered}
\frac{1}{a} \Psi_{1}^{\prime \prime}+E \alpha_{k}^{2} \omega_{1}^{\prime \prime}-\frac{\nu E n^{2} \alpha_{k}^{2}}{a^{2}} \omega_{1}=F_{1}(x ; k), \\
\Psi_{1}^{(4)}-\frac{2 n^{2}}{a^{2}} \Psi_{1}^{\prime \prime}+\frac{n^{4}}{a^{4}} \Psi_{1}-\frac{E}{a} \omega_{1}^{\prime \prime}=F_{2}(x ; k),
\end{gathered}
$$

where

$$
\begin{gathered}
F_{1}(x ; k)=-2 E \alpha_{k} \omega_{0}^{\prime \prime}-\frac{n^{2}}{a^{3} \alpha_{k}^{2}}\left(2 \nu \alpha_{k}+f_{1}(k)\right) \frac{\cos \left(\frac{x}{\alpha_{k} a}\right)}{\cos \left(\frac{l}{\alpha_{k} a}\right)} \Psi_{0} \\
-\frac{E n^{2}}{a^{2}}\left(f_{1}(k)-\left(2 \nu \alpha_{k}+f_{1}(k)\right)\right) \frac{\cos \left(\frac{x}{\alpha_{k} a}\right)}{\cos \left(\frac{l}{\alpha_{k} a}\right)} \omega_{0}, \\
F_{2}(x ; k)=\frac{E n^{2}}{a^{3} \alpha_{k}^{2}}\left(2 \nu \alpha_{k}+f_{1}(k)\right) \frac{\cos \left(\frac{x}{\alpha_{k} a}\right)}{\cos \left(\frac{l}{\alpha_{k} a}\right)} \omega_{0} .
\end{gathered}
$$

The function $\omega_{1}$ can be eliminated between (4.24a) and (4.24b) to obtain

$$
\begin{aligned}
\Psi_{1}^{(6)}+\frac{1}{a^{2}}\left(\frac{1}{\alpha_{k}^{2}}-(2+\nu) n^{2}\right) & \Psi_{1}^{(4)}+\frac{(1+2 \nu) n^{4}}{a^{4}} \Psi_{1}^{\prime \prime} \\
& -\frac{\nu n^{6}}{a^{6}} \Psi_{1}=\frac{1}{a \alpha_{k}^{2}} F_{1}^{\prime \prime}+F_{2}^{\prime \prime}-\frac{\nu n^{2}}{a^{2}} F_{2},
\end{aligned}
$$


where $\Psi_{1}(x ; k)$ is to satisfy the boundary conditions (4.10) and

$$
\Psi_{1}^{(5)}(0 ; k)=\Psi_{1}^{(4)}(l ; k)=0 .
$$

The functions $F_{1}(x ; k)$ and $F_{2}(x ; k)$ can be written out explicitly. They are

$$
\begin{array}{r}
F_{1}(x ; k)=\left(\frac{-E \beta_{k}^{2}}{a \alpha_{k}^{2}\left(\beta_{k}^{2}+\nu n^{2} \gamma^{2}\right)}\left(2 \alpha_{k} \beta_{k}^{2}-n^{2} \gamma^{2} f_{1}(k)\right) \cos \left(\beta_{k} \frac{x}{l}\right)\right) \\
-\frac{E \nu \gamma^{4} n^{4}\left(2 \nu \alpha_{k}+f_{1}(k)\right)}{a \alpha_{k}^{2}\left(\beta_{k}^{2}+\nu n^{2} \gamma^{2}\right) \cos \left(\frac{l}{\alpha_{k} a}\right)} \cos \left(\frac{x}{\alpha_{k} a}\right) \cos \left(\beta_{k} \frac{x}{l}\right), \\
F_{2}(x ; k)=\frac{-E n^{2} \gamma^{2} \beta_{k}^{2}\left(2 \nu \alpha_{k}+f_{1}(k)\right)}{a^{2} \alpha_{k}^{4}\left(\beta_{k}^{2}+\nu n^{2} \gamma^{2}\right) \cos \left(\frac{l}{\alpha_{k} a}\right)} \cos \left(\frac{x}{\alpha_{k} a}\right) \cos \left(\beta_{k} \frac{x}{l}\right) .
\end{array}
$$

In calculating (4.25) we have used (4.14), (4.16) and the fact that

$$
\begin{gathered}
A_{k}=E l^{2}, \\
B_{k}=\frac{-a \gamma^{2} \beta_{k}^{2}}{\alpha_{k}^{2}\left(\beta_{k}^{2}+\nu n^{2} \gamma^{2}\right)}
\end{gathered}
$$

is a solution of (4.18).

The equation (4.26) with the homogeneous boundary conditions (4.10) and (4.27) does not have a solution for all values of $f_{1}(k)$. Indeed the Fredholm theorem [4] implies that a necessary and sufficient condition for a solution is that

$$
\int_{0}^{l}\left(\frac{1}{a \alpha_{k}^{2}} F_{1}^{\prime \prime}+F_{2}^{\prime \prime}-\frac{\nu n^{2}}{a^{2}} F_{2}\right) \cos \left(\frac{\beta_{k} x}{l}\right) d x=0 .
$$

Equation (4.30) is an equation for the determination of $f_{1}(k)$. After an integration by parts Equation (4.30) can be simplified to

$$
\int_{0}^{l}\left(\frac{\beta_{k}^{2}}{a \alpha_{k}^{2}} F_{1}+\left(\beta_{k}^{2}+v_{n}^{2} \gamma^{2}\right) F_{2}\right) \cos \left(\beta_{k} \frac{x}{l}\right) d x=0 .
$$

After performing the integration indicated in (4.31) we can solve for $f_{1}(k)$. The result can be written

$$
f_{1}(k)=\frac{\alpha_{k} \cos \frac{\gamma}{\alpha_{k}}-\frac{4 n^{2} \nu \gamma \alpha_{k}^{4}}{\gamma^{2}-4 \beta_{k}^{2} \alpha_{k}^{2}}\left(1+\frac{2 \nu n^{2} \gamma^{2}}{\beta_{k}^{2}}\right) \sin \frac{\gamma}{\alpha_{k}}}{\frac{n^{2} \gamma^{2}}{2 \beta_{k}^{2}} \cos \frac{\gamma}{\alpha_{k}}+\frac{2 n^{2} \gamma \alpha_{k}^{3}}{\gamma^{2}-4 \beta_{k}^{2} \alpha_{k}^{2}}\left(1+\frac{2 \nu n^{2} \gamma^{2}}{\beta_{k}^{2}}\right) \sin \frac{\gamma}{\alpha_{k}}} .
$$


The case we wish to study in detail is the case where $\alpha_{k} \ll 1\left(\beta_{k} \gg 1\right)$, i.e., the small stress case. It is a consequence of (4.16) that

$$
\begin{aligned}
& \alpha_{k}=\frac{\gamma}{\beta_{k}}-\frac{(2+\nu) n^{2} \gamma^{3}}{2 \beta_{k}^{3}}+\mathcal{O}\left(\frac{1}{\beta_{k}^{5}}\right), \\
& \frac{\gamma}{\alpha_{k}}=\beta_{k}+\frac{(2+\nu)}{2} \frac{n^{2} \gamma^{2}}{\beta_{k}}+\mathcal{O}\left(\frac{1}{\beta_{k}^{3}}\right) .
\end{aligned}
$$

In addition it follows from (4.33) that

$$
\begin{aligned}
\cos \frac{\gamma}{\alpha_{k}} & =-(-1)^{k} \sin \left[\frac{(2+\nu)}{2} \frac{n^{2} \gamma^{2}}{\beta_{k}}+\mathcal{O}\left(\frac{1}{\beta_{k}^{3}}\right)\right] \\
& =-(-1)^{k}\left[\frac{2+\nu}{2} \frac{n^{2} \gamma^{2}}{\beta_{k}}\right]+\mathcal{O}\left(\frac{1}{\beta_{k}^{3}}\right), \\
\sin \frac{\gamma}{\alpha_{k}} & =(-1)^{k} \cos \left[\frac{2+\nu}{2} \frac{n^{2} \gamma^{2}}{\beta_{k}}+\mathcal{O}\left(\frac{1}{\beta_{k}^{3}}\right)\right] \\
& =(-1)^{k}\left[1-\frac{(2+\nu)^{2}}{8} \frac{n^{4} \gamma^{4}}{\beta_{k}^{2}}\right]+\mathcal{O}\left(\frac{1}{\beta_{k}^{4}}\right) .
\end{aligned}
$$

Equations (4.33) and (4.34) can be used to evaluate $f_{1}(k)$ for large values of $k$. In particular, we find

$$
f_{1}(k)=\frac{(2+\nu) \gamma}{\left(\frac{4}{3}+\frac{(2+\nu)}{2} n^{2} \gamma^{2}\right)} \beta_{k}+\mathcal{O}\left(\frac{1}{\beta_{k}}\right) .
$$

We will use (4.21) with $f_{1}(k)$ given by (4.35) to approximate the solution of (4.20) with $P=0$. This approximation should be valid for small $\alpha$ (i.e., large $k$ ). We find that

$$
\alpha(k)=\alpha_{k}+\frac{\nu \alpha_{k}}{f_{1}(k)} .
$$

Using (4.33) and (4.35) we find

$$
\alpha(k)=\frac{\gamma}{\beta_{k}}+\left(\frac{4 \nu}{3(2+\nu)}-n^{2} \gamma^{2}\right) \frac{\gamma}{\beta_{k}^{3}}+\mathcal{O}\left(\frac{1}{\beta_{k}^{5}}\right) .
$$

Equation (4.37) gives the desired approximation to the eigenvalues of (4.9).

It is possible to solve (4.26) exactly. However, the computations are extremely tedious and the actual value of the coefficients of terms is the solution and of less importance than the terms themselves. In order to find the terms which occur in the solution of (4.26) we first find a complete set of solutions for the homogeneous version of (4.26). Such a set can be found exactly. They are $\cos \left(\beta_{k} x / l\right), \sin \left(\beta_{k} x / l\right), \cos \lambda_{1} x, \sin \lambda_{1} x, \cosh \lambda_{2} x, \sinh \lambda_{2} x$ 
where

$$
\begin{aligned}
& \lambda_{1,2}^{2}=\left\{\left[\left(\frac{(1+2 \nu) n^{4} \gamma^{2}}{\beta_{k}^{2}}\right.\right.\right.\left.\left.+\frac{\nu n^{6} \gamma^{4}}{\beta_{k}^{4}}\right)^{2}+\frac{4 \nu n^{6} \gamma^{2}}{\beta_{k}^{2}}\right]^{1 / 2} \\
&\left.\mp\left[\frac{(1+2 \nu) n^{4} \gamma^{2}}{\beta_{k}^{2}}+\frac{\nu n^{6} \gamma^{4}}{\beta_{k}^{4}}\right]\right\} / 2 a^{2} .
\end{aligned}
$$

For large $k$ equation (4.38) implies

$$
\lambda_{1,2}=\frac{\nu^{1 / 4} n^{3 / 2} \gamma^{1 / 2}}{a \beta_{k}^{1 / 2}} \mp \frac{(1+2 \nu) n^{5 / 2} \gamma^{3 / 2}}{4 a \nu^{1 / 4} \beta_{k}^{3 / 2}}+\mathcal{O}\left(\frac{1}{\beta_{k}^{5 / 2}}\right) .
$$

There is no difficulty in showing that the solution of (4.26) satisfying the boundary conditions has the form

$$
\begin{aligned}
\Psi_{1}=C_{1} \cos \lambda_{1} x & +C_{2} \cosh \lambda_{2} x+C_{3} x \sin \left(\beta_{k} \frac{x}{l}\right) \\
& +C_{4} \cos \left(\frac{\gamma}{\alpha_{k}}+\beta_{k}\right) \frac{x}{l}+C_{5} \cos \left(\frac{\gamma}{\alpha_{k}}-\beta_{k}\right) \frac{x}{l} .
\end{aligned}
$$

If $f_{1}(k)$ satisfies (4.31) (or equivalently (4.32)) the coefficients $C_{i}$ in (4.40) are uniquely determined. Thus when $k$ is sufficiently large the solution is a combination of highly oscillatory terms and nonoscillatory terms $\left(\cos \lambda_{1} x, \cosh \lambda_{2} x\right)$.

\section{REFERENCES}

[1] T. von Karman and H. S. Tsien, The buckling of thin cylindrical shells under axial compression, Jour. Aero. Sci. 7, 303 (1939)

[2] E. Bromberg and J. J. Stoker, Non-linear theory of curved elastic sheets, Quart. Appl. Math. 3, 246 (1945/46)

[3] E. Reissner, Rotationally symmetric problems in the theory of thin elastic shells, 3rd U.S. Natn. Congr. of Applied Mechanics, 51 (1958)

[4] R. Courant and D. Hilbert, Methods of Mathematical Physics, Vol. 1, Interscience Publishers, New York (1953) 